\title{
Translation of research discoveries to clinical care in arrhythmogenic right ventricular cardiomyopathy in Newfoundland and Labrador: Lessons for health policy in genetic disease
}

\author{
Kathy Hodgkinson, PhD ${ }^{1,2}$, Elizabeth Dicks, PhD ${ }^{1}$, Sean Connors, MD, DPhil, FRCPC $C^{3}$, \\ Terry-Lynn Young, $P h D^{2}$, Patrick Parfrey, MD, FRCPC ${ }^{l}$, and Daryl Pullman, PhD ${ }^{4}$
}

\begin{abstract}
Arrhythmogenic right ventricular cardiomyopathy, a lethal autosomal dominant cause of sudden cardiac death in young people, is prevalent in Newfoundland and Labrador (genetic subtype ARVD5). In the absence of implantable cardioverter defibrillator treatment, death rates are extremely high. Research into arrhythmogenic right ventricular cardiomyopathy (ARVD5) began in the 1980s and the causative gene and mutation were discovered in 2008. The decades of research highlighted major issues associated with the ethical management of genetic information and the translation of research findings to clinical care. We describe these issues and the strategies used in managing them. Effective knowledge transfer of the research information has resulted in systematic clinical and genetic screening coupled with genetic counseling and treatment for at-risk family members. Improved survival for patients has been one clear result of this strategy. Optimal care of families where individuals are at-high risk of inheriting a disease with high morbidity and mortality requires the full integration of both genetic research and clinical genetics programs. Although yet to be fully effected in our setting, our discussion highlights both the ethical necessity as well as some practical barriers in realizing this outcome. Genet Med 2009:11(12):859-865.
\end{abstract}

Key Words: $A R V C$, research, consent process, clinical responsibility, ethics, duty to warn

G enetic research into human disease provides information pertinent to an understanding of disease pathogenesis and can influence management strategies for that disease. Certain genetic conditions arise more frequently in Newfoundland and Labrador due primarily to historic settlement patterns. ${ }^{1}$ Founder mutations are thus recognized for several autosomal dominant diseases, ${ }^{2-4}$ including arrhythmogenic right ventricular cardiomyopathy (ARVC). ARVC in Newfoundland and Labrador is a cause of sudden cardiac death (SCD) because of lethal tachyarrhythmias $^{5}$ caused primarily by a founder mutation in TMEM43 (ARVD5). ${ }^{6}$ A long-standing research project on ARVC has changed both policy and procedure in the manage-

From the ${ }^{1}$ Clinical Epidemiology Unit, Memorial University; ${ }^{2}$ Discipline of Genetics, Memorial University; ${ }^{3}$ Division of Cardiology, Memorial University; and ${ }^{4}$ Division of Community Health and Humanities, Memorial University, St. John's, Newfoundland, Canada.

Daryl Pullman, Department of Medical Ethics, Division of Community Health and Humanities, Memorial University of Newfoundland, St. John's, NL, Canada A1B 3V6. E-mail: dpullman@mun.ca.

The first two authors contributed equally to this manuscript

Disclosure: The authors declare no conflict of interest.

Submitted for publication May 13, 2009

Accepted for publication September 18, 2009.

DOI: 10.1097/GIM.0b013e3181c20bb3 ment of genetic disease because of the many lessons learned in the decades during which the study has been in progress.

ARVC is a biventricular cardiomyopathy characterized by fibro-fatty infiltration of the myocardium, which can lead to ventricular tachyarrhythmias and SCD. A positive ARVC diagnosis depends on the number of clinical test abnormalities (major and/or minor criteria) exhibited by the individual. ${ }^{7}$ However, clinical diagnosis is difficult and SCD may be the first presentation. ${ }^{5}$ Many of the criteria require tertiary level clinical testing, and the presence of ARVC pathology at autopsy in the absence of other abnormal clinical test results does not fulfill the diagnostic criteria. The diagnostic utility of these criteria for diagnosis of ARVC, especially in the early stages, is poor.

ARVC is almost always inherited as an autosomal dominant disorder. Clinical diagnostic difficulties are compounded by other factors common to all genetic disorders including variable expressivity (carriers may present with different signs and symptoms) and reduced penetrance (some carriers remain asymptomatic). Hence, a genetic test able to provide a precise presymptomatic diagnosis unrelated to the utility of clinical testing obviates many of the diagnostic difficulties.

As early as the 1980s, individuals with ARVC in Newfoundland participated in research designed to elucidate etiology. The most recent study that began in 1997, aimed to define the natural history and phenotypic expression of ARVC and to find the gene responsible. Linkage to Chromosome 3 was published in $1998^{8}$ and a disease-associated founder haplotype (ARVD5) - a series of linked genetic markers surrounding the gene locus, which are inherited together in affected individuals across generations ${ }^{9}$ - was recognized. In 2007, the local research team discovered the causative mutation in a novel gene TMEM43 within the disease-associated founder haplotype. ${ }^{6}$ To date, 17 families have been identified with a founder mutation in TMEM43 on Chromosome 3 (ARVD5). All subjects born at an a priori $50 \%$ pedigree risk have been identified, all medical records obtained (when available), and all relevant cardiac outcomes have been analyzed.

This research demonstrated that this genetic subtype of ARVC is lethal, with a median age to death in men of 41 years compared with 71 years in women. ${ }^{5}$ Despite the sex influence, the disease follows a classic autosomal dominant mode of inheritance with clear male-to-male transmission (Fig. 1). Our study determined that the disease was $100 \%$ penetrant (as defined by clinical sign or symptom) in both sexes (by age 63 years and 76 years in men and women, respectively), and heart failure was a later manifestation in survivors of SCD. ${ }^{6}$ Between 1998 and 2007, before the identification of the causative mutation, presymptomatic diagnosis of carriers was possible by utilizing "research generated" haplotype analysis. This allowed for prophylactic use of implantable cardioverter defibrillators 


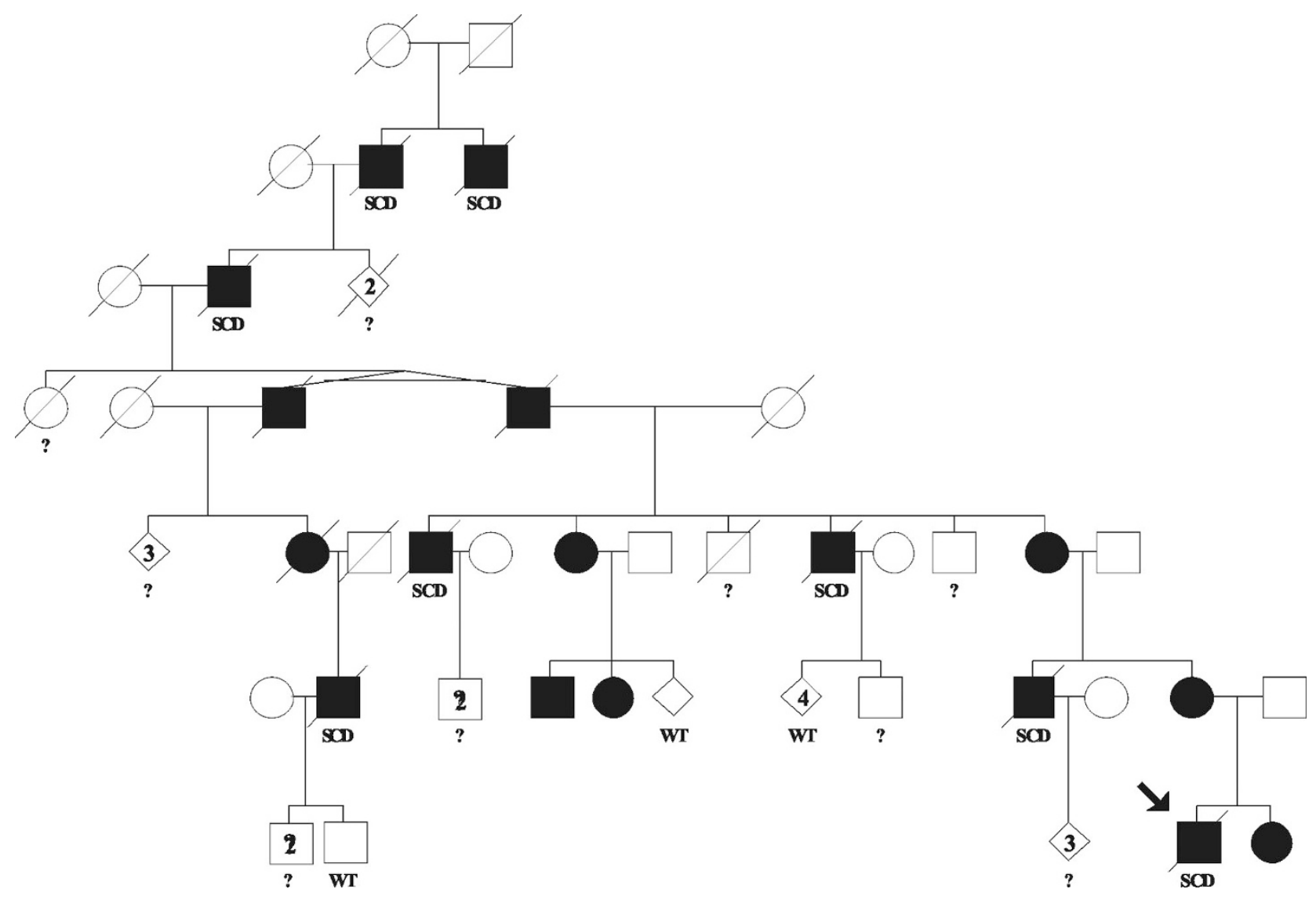

Fig. 1. An ARVC family pedigree showing autosomal dominant inheritance. Squares $=$ males, Circles $=$ females, Blacked in symbol = ARVD5 (positive diagnosis based on SCD under 50 years, cardioversion for ventricular tachycardia or ventricular fibrillation under 50 years, obligate carrier status, and/or the presence of the TMEM43 S358L mutation). Oblique stroke through symbol = dead, WT = wild type for TMEM43, Question mark (?) = remain at an a-priori 50\% risk, Arrow $=$ proband.

(ICD). A subsequent analysis indicated that the ICD significantly improved survival of men in this population. ${ }^{5}$

The significant morbidity and mortality exhibited by this disease, the high penetrance, and the high risk to relatives led to a number of challenging issues with regard to the management of these patients, their families, and the data generated both through research and clinical means. Issues included: (i) the ethical management of genetic information and the translation of research findings to clinical care; (ii) the process of technology transfer from the research laboratory to the clinical laboratory; and (iii) the optimal management of genetic data whether generated through research or clinically. In what follows we highlight key events in the history of the research, discuss lessons learned from the experience, and outline some continuing challenges in the on-going clinical management of these families.

This article highlights some aspects of the journey taken by the researchers, clinicians, ethicists, and policy makers who have worked on ARVD5 in Newfoundland during the past two decades. Figure 2 catalogues some of the key events and the lessons learned during the search for the causative mutation for ARVC in Newfoundland and Labrador, and the on-going management of patients and families during that time. We believe our experiences will prove instructive for all genetic research dealing with genetic conditions with high morbidity and mortality and for their effective clinical management.
The ethical management of genetic information and the translation of research findings to clinical care

\section{Lesson 1: The need for a centralized biobank to preserve valuable samples over time}

Following the publication in 1980 of results that linked hypertrophic cardiomyopathy with specific human lymphocyte antigen types, ${ }^{10}$ researchers in Newfoundland were interested in exploring the relationship between ARVC and human lymphocyte antigen. The recognition of a large ARVC family led to the treatment of an affected male in Ontario, ${ }^{11}$ followed by the first description of the ARVC family by the research team in $1988 .^{12}$

Genetic studies involving large groups of people, in which accurate phenotyping and the creation of a comprehensive family pedigree are central to success, are slow, and cumbersome by nature. The data ultimately derived are priceless. The initial samples were seen as resources related exclusively to one research project designed to answer one research question. Archiving was not available or desired at the time of the initial study, nor was it common practice to initiate contact with clinical genetics services.

Collected blood samples from this initial research were stored until space limitations resulted in their destruction in the early 1990s. By the time the subsequent study that eventually led to the identification of the causative mutation began in 1997 , 


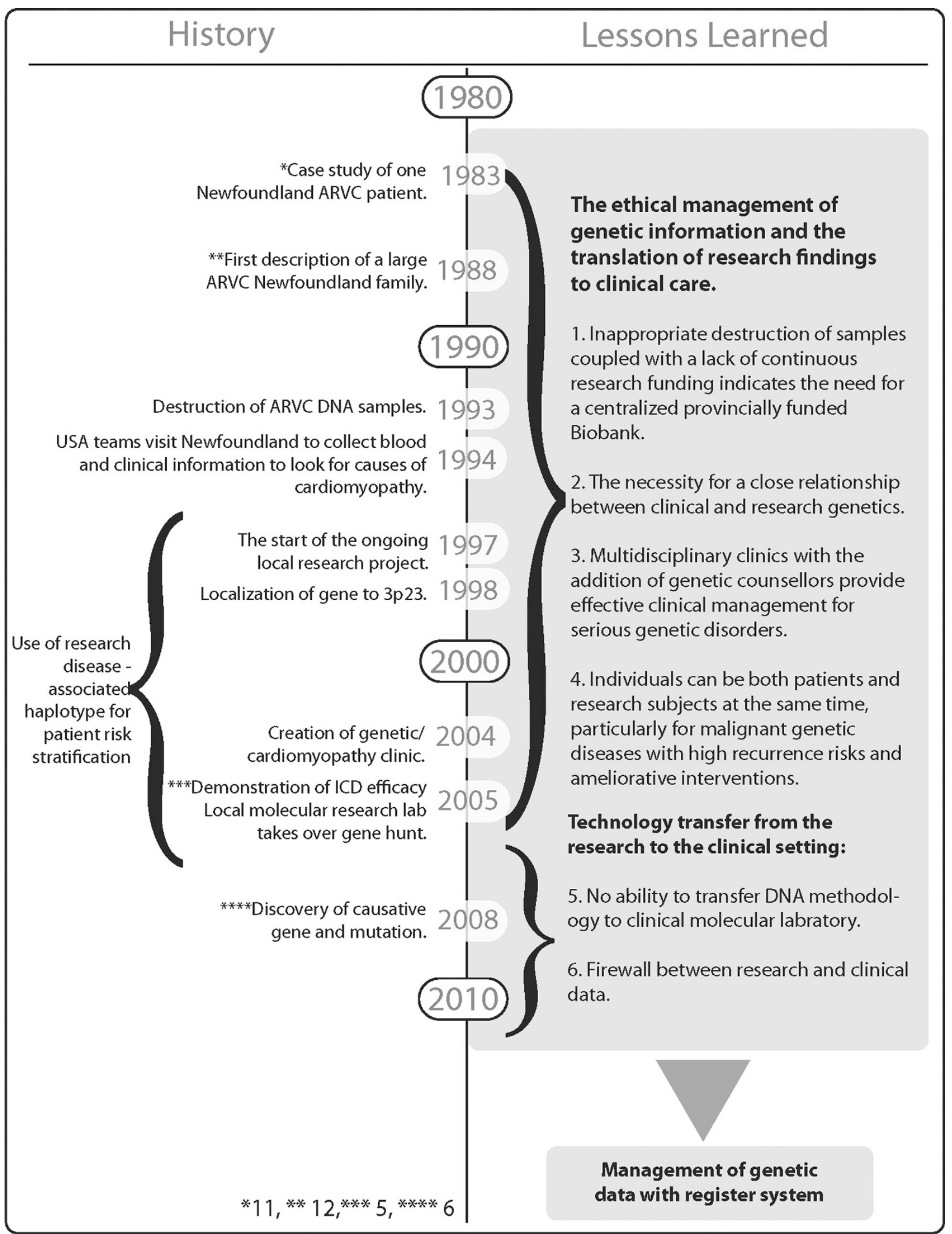

Fig. 2. Temporal sequence of the history of the ARVC project and the lessons learned. 
many of the original family members had died and were thus no longer available to provide DNA. This was a catastrophic loss as affected individuals in these large families were often defined by SCD at a young age. DNA is usually not available from deceased subjects or is fragmented and thus of limited use when obtained from a fixed tissue sample. This reinforces the need to treat biological samples as an irreplaceable resource and to maintain these samples. This is particularly true when the disease is genetic, has high morbidity and mortality, and when many questions with regard to the underlying pathophysiology remain to be determined. It is a sobering thought that the gene for ARVD5 may have been found much sooner had DNA samples been available from those who had subsequently died. Such storage and retention problems would be obviated with a central biobank for the storage of all DNA samples from families with genetic disease.

\section{Lesson 2: The necessity for a close relationship between clinical and research genetics}

The DNA that was used to define the locus for ARVD5 ${ }^{8}$ had been collected by US investigators during the 1990s. A diseaseassociated DNA haplotype made it possible to define those at extremely high risk of having the as yet unidentified ARVD5 gene. However, the US researchers resisted sharing what they defined as "research data," with the Newfoundland clinical genetics program. These research data included clinical test results in the form of 12 lead electrocardiography and echocardiograms. The absence of this information resulted in a delay in translating these findings into patient care. Following subsequent review of the US data (provided many years later), it was discovered that in some cases this delay had catastrophic consequences. This in turn prompted a broader discussion of the nebulous distinction between research data and clinical information when dealing with conditions like ARVD5. ${ }^{13}$

This untoward experience was the stimulus to establish an international genetic cardiomyopathy research group in St. John's. The team included members of the clinical genetics program as well as collaborators in Berlin and London. The goal was to expedite the search for the causative mutation. The disease-associated haplotype analysis for family members identified initially by the US team was recapitulated in 1998 by Dr. Ludwig Thierfelder's laboratory in Berlin and subsequently in 2005 by Dr. Terry Lynn Young's laboratory in Newfoundland. Linkage results were provided to the clinical research team (Fig. 2). "High-risk" and "low-risk" subjects were defined on the basis of those who had either inherited or not inherited the disease-associated haplotype, respectively.

Due to meiotic recombination events resulting in the inheritance of a partial haplotype, some individuals remained at $50 \%$ pedigree risk. These individuals, and those with a 'low risk' research result, could not therefore be discharged from clinical follow-up. Clinical testing on an annual basis was thus required. As the earliest confirmed SCD in these families was 19 years for men and 37 years for women, ${ }^{5}$ it was evident that deaths would occur unless the research team provided presymptomatic help in the form of a DNA haplotype and arranged clinical management for family members. This placed an enormous burden on the research laboratories because clinical decisions had to be made based on the research disease-associated haplotype. However, the tightly linked haplotype meant that even when potential sample error was factored into the equation, the likelihood of having the causative gene given the haplotype was approximately $98 \%$. Thus, this research result provided a far greater sensitivity than any single clinical test available. Although a research computer database allowed for effective tracking of the expanded pedigrees, it was impossible for one person to provide clinical care and appropriate follow-up while collecting and analyzing research data. In the meantime, pedigree analysis confirmed that many young males had died without ever visiting a doctor. Hence, based on the presence of the disease-associated haplotype, ICD therapy was offered to young asymptomatic adult males.

\section{Lesson 3: Clinical management is effective with a multidisciplinary clinic}

Once the malignant phenotype and the effectiveness of prophylactic treatment of carriers with the ICD had been established, ${ }^{5}$ a cardiac genetics clinic was created in 2004 (Fig. 2). The clinic was managed by the Eastern Health Regional Health Authority of Newfoundland and Labrador with the goal of integrating on-going research discoveries with the clinical management of these individuals. All families (160 to date) with a cardiomyopathy or inherited form of arrhythmia, including ARVC, were referred to the program to provide extensive clinical assessment and to record extended family histories. Families in the research program were formally transferred to the interdisciplinary clinic that was staffed by experienced health care providers. The clinical team included a half-time clinical genetics counselor, a half-time cardiac nurse, a cardiologist and several support staff, all under the direction of an electrophysiologist. A clinical ethicist was an invaluable team member, and several times engaged the hospital clinical ethics service to advise on issues of confidentiality, duty to warn, and so forth. ${ }^{13}$ Continued ascertainment and screening of at-risk individuals with appropriate treatment of affected individuals were a priority.

With the establishment of the clinic, the team was able to achieve comprehensive follow-up, continuity of care by established experts, streamlining of all necessary investigations, follow-up and interventions, and assurance that all individuals were treated according to established protocols. The close association between the research team and the clinicians was maintained in that the clinic used data from the molecular genetic research laboratory to identify carriers. Phenotypic data from multiple generations and subjects that had been analyzed by the research group were used to inform family members. The research genetic counselor provided the key connection between the research and the clinical teams. This was the process from the time that the haplotype was determined (1998) to the time that the mutation was defined (2008).

An analysis of the clinical data associated with ICD treatment in patients with ARVD5 indicates that this is the treatment of choice for this condition. ${ }^{5}$ At the time of writing, 104 adult ARVD5 TMEM43 mutation carriers have been offered an ICD, of which seven women and two men refused treatment. Both men subsequently died after an arrhythmia. Despite known complications associated with ICD surgery, including pocket infection hematoma or seroma, pulmonary effusion or pneumothorax, and potential lead fracture, we have had no serious complications with the ARVD5 ICD recipients in Newfoundland.

We plan to formally assess the impact of this clinic over time. In so doing, we will focus on three specific areas: (i) an evaluation of the clinical course after ICD to assess long-term morbidity and mortality rates; (ii) a monitoring of wait times to receive an ICD and for receipt of mutation results; and (iii) an assessment of psychological issues surrounding the ICD and possible sequelae. 


\section{Lesson 4: Recognize situations when individuals must be treated as both research subjects and as patients}

Participants in genetic research studies are routinely assured that their genetic information will be kept confidential. However, honoring this commitment can result in withholding critical information from those responsible for clinical follow-up of at-risk individuals. At the start of the 1997 Newfoundland ARVC study to determine the causative gene, the research ethics board (REB) approved a consent form that asked individuals to choose whether they wished to hear their research results. However, refusals to receive information became problematic for both researchers and clinicians involved with the ARVC study, particularly when a "high-risk" haplotype was confirmed by the research laboratory. In one instance, this resulted in the preventable death of a 31-year-old man who refused to hear his result, and in another, the possibility existed that an individual would continue to work in a public transport position, which would place the general public at risk (Appendix).

Given the extremely high risk of SCD and the availability of a proven prophylactic intervention, a revised consent form was approved by the local REB after many months of discussion. Potential study participants were informed that they would be given their molecular genetic results when available. Those who chose not to receive any genetic information would not have their blood drawn and hence would not participate in the research. ${ }^{13}$

The issues highlighted also had an impact on first-degree relatives to study participants. If a study subject was determined to have the disease-associated founder haplotype, all their children would be at a $50 \%$ risk. This potentially broadened the burden of information provision from the research team to the extended family. This raised the question of a potential "duty to warn" individuals or extended family members, irrespective of any implied or expressed promise of confidentiality in their original research consent. Recent literature on the ethical issues of managing genetic information for diseases with significant health issues for third parties indicates the emergence of a duty to warn on the part of physicians and perhaps researchers who are party to this information. ${ }^{14} \mathrm{We}$ have argued that the merits of this approach must reflect the severity of the condition, the recurrence risk, and the possibility of amelioration. ${ }^{13}$

\section{The process of technology transfer}

\section{Lesson 5: The need for an effective and efficient means by which to transfer DNA methodology to the clinical molecular laboratory}

Before the discovery that a missense mutation in the TMEM43 gene causes ARVD5, the genetic risk status of each individual at risk, across 15 ARVD5-linked families, was provided by the two university-based research laboratories involved in the gene hunt. The large number of at-risk individuals and the ability to save lives in gene carriers with an implantable ICD created an opportunity for the clinic to assess more accurately who should receive an ICD, and concomitantly, a challenge for the research laboratories to deliver this test. Research laboratories rely on trainees (undergraduates up to postdoctoral fellows) to perform the experimental work. However, funding from the public purse is targeted toward research, not the provision of medical services. This presents issues with regard to quality control as the standards can vary significantly between a research laboratory where training is an integral part of the mandate, and a clinical laboratory where a level of expertise is already assumed, and the quality of the clinical test result is the priority. This is not to say that research laboratory standards are always less than a clinical laboratory: sometimes the opposite may be true. A research laboratory, however, is not regulated. These challenges are magnified by the very nature of the genetic "test," which was in reality up to 17 separate "tests" or genotypes that were run, and then combined, often with genotypes on close family members, to construct a "risk haplotype." These issues were further compounded in the present case in that the laboratory received urgent requests for haplotype results at regular intervals, sometimes several in 1 day. These arose primarily from the local clinical care team, but on many occasions also from health care teams in mainland Canada and elsewhere.

After the discovery that a mutation in TMEM43 causes ARVD5, the genetic risk status can be readily assessed by a single assay and direct sequencing to check for the presence of the founder mutation. However, this test continues to be available locally only in the gene discovery laboratory (St. John's), as it is not yet available through the molecular diagnostic laboratory. In Canada, the decision to set up a genetic test and then offer it is at the discretion of the Director of the diagnostic laboratory. Thus, the transfer of a genetic test to provide both presymptomatic and diagnostic testing is not a foregone conclusion, even with frequent and lethal dominant diseases like ARVC. Nevertheless, this test is currently available in other laboratories in Canada, the United States, and Europe. This has lead to the ironic situation that the Newfoundland health care system must pay an outside clinical laboratory for a test result based on a causative mutation found by its own researchers.

Issues related to the use of genetic research results for clinical purposes and the "duty to warn" have been addressed elsewhere. ${ }^{13}$ For research purposes, we are aware of the special privacy concerns related to the sharing of personal health information in general and genetic information in particular. Such issues have been raised in the United States, ${ }^{15,16}$ and we have contributed to the discussion in Canada. ${ }^{17}$ Health information privacy legislation is pending in our province, and it provides for some flexibility with regard to the management of genetic information. ${ }^{18}$ The familial nature of genetic information presents special challenges in this regard, which are beyond the scope of the present discussion. Suffice it to say that, in our view, progressive legislation with regard to genetic privacy must strike a balance between individual protection and the broader familial and public good.

\section{Lesson 6: Optimal management of research and clinical data requires the creation of a genetic registry}

In this study, there was substantial overlap between the clinical management of at-risk family members and the ongoing research. The clinical management included genetic counseling and annual clinical cardiac screening for those at 50\% risk. Initially this latter point also included those given a "low-risk" haplotype in an attempt to exclude any remaining error. The research genetic counselor provided genetic counseling and organized cardiology screening to many at-risk individuals from 1997 to 2004 . Without this clinical involvement, deaths would have occurred. However, transfer of the pedigrees and the vast amounts of clinical data obtained on family members to the combined genetics cardiac clinic was not seamless because a central repository for genetic information was not available. Furthermore, the incorporation of new observations made by either the clinic or the research program was problematic because a firewall existed between the pedigrees and other data obtained by the research team (maintained on the University 
computers) and the clinical program (maintained on the health care system computers). This situation almost certainly exists elsewhere in Canada and the United States.

Clinical data can be collected in both institutional (e.g., hospital clinic) and community settings, under either clinical or research protocols, and by a variety of health professionals including genetic researchers, physicians, genetic counselors, research nurses, and others. The lack of coordinated data management between the teams and/or personnel can lead to suboptimal clinical management and unnecessary delays in research. It can also result in frustration among family members who are often asked to provide the same information to different health care professionals on numerous occasions.

In 1972, the World Health Organization ${ }^{19}$ recommended that medical genetics centers establish registries of genetically determined disease. The aim was to improve clinical care, to provide accurate risk assessment, and to facilitate the provision of information to families and patients. In addition, it was recognized that adequate information on aspects of many genetic conditions was lacking, so that registries could facilitate research. Many UK genetics departments have successfully used this methodology over the years, ${ }^{20-25}$ but they are much less common in North America. Such registries ensure that genetic counseling is offered to all those at risk and that appropriate clinical care is implemented. We are in the process of establishing such a register for our ongoing work in Newfoundland and Labrador.

A genetic registry will allow individual and associated familial and clinical information to be housed in one place, thus enabling both researchers and clinicians to access all relevant information in a timely manner for their various purposes. In relation to the Mendelian cardiac disorders with an increased risk of SCD, we propose that all family members be entered in a centralized genetic register (database), where each individual is attached to a digital pedigree, and is part of a clinical genetics program. During the initial work up by a genetic counselor, prospective participants will be informed that they will be enrolled on the register to maintain contact and to provide for clinical follow-up, unless they choose otherwise. In addition, being in the registry would mean that researchers might contact individuals to invite them to participate in research projects. Individuals could opt out of each research project in which they are asked to participate, or opt out of any subsequent contact by any research team whatsoever. Researchers would require approval from the relevant REB before accessing the database. Depending on the nature of the research study, either anonymized or identifiable data would be available. The latter case would occur only with the consent of the individuals involved. Relevant research findings would be exchanged with the clinical group to facilitate appropriate clinical follow-up. Clinical genetics staff, in turn, would have access to the clinical database. Other specialists could liaise with genetics staff for information necessary to the optimal management of their patients. Any changes to family history information, whether generated through a research study or through clinical contact, would be made to a centralized pedigree, and a limited number of staff would be authorized to make such changes. Thus, only one comprehensive family tree would be available.

Managing the data in this manner will contribute to better clinical management of patients and family members as they will have access to clinical screening, testing, and treatment when appropriate. Clinicians will be able to access relevant research results as soon as they become available. Although the level of staffing for a register system is high, we are hopeful that the benefits in terms of prevention of adverse health outcomes will more than compensate for the up front costs. ${ }^{26}$

\section{CONCLUSIONS}

Newfoundland's ARVC SCD "riddle" was solved by an interdisciplinary team comprising clinicians, researchers, genetic counselors, nurses, molecular biologists, and ethicists. The ARVC problem was identified in terms of the patients, the families, and their communities. These stake holders combined with both research and clinical staff to ensure that the research knowledge generated was translated into effective patient care. Initial failure to provide effective and efficient multidisciplinary care occurred, but the initiation of a cardiac genetics clinic integrated with the research group was successful in delivering appropriate services to the families. Ethical issues regarding the use of research data have arisen, and measures to deal with some of these tensions have been initiated. Technology transfer of the novel gene discovery to the clinical molecular genetics laboratory and integration of research data with clinical care has been problematic, but the process has provided useful lessons in how to deal with future projects.

The lessons learned have resulted in a more efficient system for dealing with ARVC in particular, and this wisdom should be transferable to other genetic diseases. The major lesson learned emphasizes the need for a systematic process that will ensure the timely and effective translation of genetic research discoveries for optimal clinical care. For families segregating a potentially lethal mutation with a high recurrence risk and penetrance, particularly when ameliorative intervention is possible, full integration of both the genetic research and clinical genetics programs is required.

\section{ACKNOWLEDGMENTS}

This project was supported by Genome Canada, The Janeway Children's Hospital Foundation, and St. Jude Medical through AMGGI. Elizabeth Dicks was supported by APOGEE-Net Agence d'évaluation des technologies et des modes d'intervention en santé (AETMIS). Special thanks to Ms. Carol Tilley BN, RN, for assistance within the cardiology program. Jennifer Armstrong provided invaluable assistance with graphics.

\section{REFERENCES}

1. Mannion J, editor. The peopling of Newfoundland: essays in historical geography. St. John's: Memorial University, 1977

2. Dicks E, Ravani P, Langman D, Davidson W, Pei Y, Parfrey P. Incident renal events and risk factors in autosomal dominant polycystic kidney disease: a population and family-based cohort followed for 22 years. Clin $J$ Am Soc Nephrol 2006;1:710-717.

3. Olufemi S, Green J, Manickam P, et al. Common ancestral mutation in the MEN-1 gene is likely responsible for the prolactinoma variant of MEN1 (MEN1 burin) in four kindreds from Newfoundland. Hum Mutat 1998;11: 264-269.

4. Stuckless S, Parfrey P, Woods M, et al. The phenotypic expression of three MSH2 mutations in large Newfoundland families with Lynch syndrome. Fam Cancer 2007;6:1-12.

5. Hodgkinson K, Parfrey P, Bassett A, et al. The impact of implantable cardioverter defibrillator therapy on survival in autosomal dominant arrhythmogenic right ventricular cardiomyopathy (ARVD5). J Am Coll Cardiol 2005;45:400-408

6. Merner ND, Hodgkinson KA, Haywood AF, et al. Arrhythmogenic right ventricular cardiomyopathy type 5 (ARVD5) is a fully penetrant, lethal arrhythmic disorder caused by a missense mutation in the TMEM43 gene. Am J Hum Genet 2008;82:809-821.

7. McKenna WJ, Thiene G, Nava A, et al. Diagnosis of arrhythmogenic right ventricular dysplasia/cardiomyopathy. Task Force of the Working Group Myocardial and Pericardial disease of the European Society of Cardiology and the Scientific Council on Cardiomyopathies of the International Society and Federation of Cardiology. Br Heart J 1994;71:215-218. 
8. Ahmad F, Li D, Karibe A, et al. Localisation of a gene responsible for arrhythmogenic right ventricular dysplasia to chromosome 3p23. Circulation 1998;98:2791-2795.

9. Norman M, Longley M, Seldon M, Hodgkinson K, McKenna W, Thierfelder L. A founder mutation in a Canadian cardiomyopathy population can cause a novel variant of arrhythmogenic right ventricular cardiomyopathy and dilated cardiomyopathy [Abstract]. Eur J Cardiol. In press.

10. MacArthur C, McKenna WJ. HL-A and hypertrophic cardiomyopathy. Am Heart J 1980;99:542-543.

11. Guiraudon G, Klein G, Gulaumshein S, et al. Total disconnection of right ventricular free wall: surgical treatment of right ventricular tachycardia associated with right ventricular dysplasia. Circulation 1983;67:463740 .

12. Marshall W, Furey M, Larsen B, et al. Right ventricular cardiomyopathy and sudden death in young people [letter]. $N$ Engl J Med 1988;319:174-175.

13. Pullman D, Hodgkinson K. Genetic knowledge and moral responsibility: ethical concerns at the interface of genetic research and clinical practice. Clin Genet 2006;69:199-203.

14. McAbee GN, Sherman J. Physician's duty to warn third parties about the risk of genetic diseases. Pediatrics 1998;102(1 Pt 1):140-142.

15. Kulynych J, Dorn D. The new HIPPA (Health Insurance Portability and Accountability Act of 1996) medical privacy rule: help or hindrance for clinical research? Circulation 2003;108:919-920.

16. National Academy of Sciences. Effect of the HIPPA privacy rule on health research. In: Proceedings of a workshop presented to the National Cancer Policy Forum; Washington, DC: Institute of Medicine: National Academies Press, 2006.

17. Pullman D, Buehler S, Felt L, et al. Sorry you can't have that information: data holder confusion regarding privacy requirements for personal health information and the potential chilling effect on health research. Healthcare Policy 2009; 4:61-76.

18. Bill 7: An Act to Provide for the Protection of Personal Health Information. Available at: http://www.assembly.nl.ca/buisiness/bills/Bill0807.htm; 2008.

19. WHO. Control of Hereditary diseases. WHO technical report series. 1972;497.

20. Elles RG, Hodgkinson K, Mallick NP, et al. Diagnosis of adult polycystic kidney disease by genetic markers and ultrasonographic imaging in a voluntary family register. $J$ Med Genet 1994;31:115-120.

21. Kerzin-Storrar L, Wright C, Williamson PR, et al. Comparison of genetic services with and without genetic registers: access and attitudes to genetic counselling services among relatives of genetic clinic patients. J Med Genet 2002;39:e85

22. Dean J, Fitzpatrick D, Farndon P, Kingston H, Cusine D. Genetic registers in clinical practice: a survey of UK clinical geneticists. $J$ Med Genet 2000;37:636-640
23. Maddock I, Moran A, Maher E, et al. A genetic register for von HippelLindau disease. J Med Genet 1996;33:120-127.

24. Wright C, Kerzin-Storrar L, Williamson P, et al. Comparison of genetic services with and without genetic registers: knowledge, adjustment, and attitudes about genetic counselling among probands referred to three genetic clinics. J Med Genet 2002;39:e84.

25. Harper P. Genetic registers and the prevention of inherited disorders. Proc Annu Symp Eugen Soc 1983;19:117-137.

26. Green J. Development, implementation and evaluation of clinical and genetic screening program for hereditary tumour syndrome. St. John's: Memorial University, 1995.

\section{APPENDIX}

A school bus plunges off a cliff. The 39-year-old bus driver and 36 children are dead. However, the bus driver had died before the bus left the road, killed by an inherited cardiac arrhythmia. The bus driver had participated in a genetic research project to determine the underlying gene for the inherited sudden cardiac death syndrome in his extended family. Although the genetic researchers had determined a tight linkage assignment (the putative gene mutation was still unknown), he chose not to hear his results, as was his right under research ethics guidelines. Privacy legislation ensured his results would not be shared with his physician without his consent. Had the bus driver consented to receive his research results he would have received an implantable cardioverter defibrillator. The intervention would have saved his life, and the lives of the 36 children, although it would have cost him his job.

It is highly unlikely that the parents would see this scenario as being unforeseeable and impossible to prevent.

The possibility of a scenario similar to the above was very real in our experience. The horrors implicit in this fictional account led us to consider which forms of genetic research should be indivisible from clinical care, and which should be considered separate. 